\title{
Changes in serial laboratory test results in snakebite patients: when can we safely exclude envenoming?
}

\author{
Graham Ireland, Simon G A Brown, Nicholas A Buckley, Jeff Stormer, Bart J Currie, Julian White, \\ David Spain and Geoffrey K Isbister for the Australian Snakebite Project Investigators
}

$\mathrm{T}$ he majority of patients presenting to Australian emergency departments with suspected snakebite do not develop envenoming. ${ }^{1-3}$ The accepted policy in Australia is for patients to be observed and have serial blood samples tested for up to 24 hours after a bite, as well as removal of any first aid such as pressure bandages with immobilisation (PBI). This practice has some support from anecdotal case reports of delayed envenoming, but has never been formally tested in snakebite cases. ${ }^{4-8}$ According to one study from southern Queensland, some hospitals discharge asymptomatic patients with normal blood test results 4 to 6 hours after the bite. However, the study included only 34 envenomed patients, and there are concerns about its applicability to other geographical regions. ${ }^{9}$

No Australian study has systematically examined the changes in early laboratory test results observed in envenomed or nonenvenomed patients after snakebite. Some early blood test results may be indicative of severe envenoming and therefore useful to determine if envenoming has occurred. Our aim was to determine which laboratory tests are first associated with severe envenoming after a snakebite, when (ie, how long after the bite) the test results become abnormal, and whether this information might assist in determining a safe observation period after suspected snakebite.

\section{METHODS}

\section{Setting and study design}

This was a cohort study of patients with confirmed or suspected snakebite recruited to the Australian Snakebite Project (ASP). ASP recruitment and data collection processes have previously been described in detail. ${ }^{10-12}$ In brief, ASP is a national, prospective, multicentre study that recruits adults and children (aged $>2$ years) with suspected or definite snakebite from over 100 hospitals. All patients have demographic and clinical information, laboratory test results and treatments recorded on a clinical research form, which is then entered into a purpose-built relational database.

\section{ABSTRACT}

Objectives: To determine which laboratory tests are first associated with severe envenoming after a snakebite, when (ie, how long after the bite) the test results become abnormal, and whether this can determine a safe observation period after suspected snakebite.

Design, patients and setting: Prospective cohort study of 478 patients with suspected or confirmed snakebite recruited to the Australian Snakebite Project from January 2002 to April 2009, who had at least three sets of laboratory test results and at least 12 hours of observation in hospital after the bite. Severe envenoming was defined as venominduced consumption coagulopathy (VICC), myotoxicity, neurotoxicity or thrombotic microangiopathy.

Main outcome measures: International normalised ratio (INR), activated partial thromboplastin time (aPTT), creatine kinase (CK) level, and neurological examination.

Results: There were 240 patients with severe envenoming, 75 with minor envenoming and 163 non-envenomed patients. Of 206 patients with VICC, 178 had an INR > 1.2 (abnormal) on admission, and the remaining 28 had an INR $>1.2$ within 12 hours of the bite. Of 33 patients with myotoxicity, a combination of CK $>250 \mathrm{U} / \mathrm{L}$ and an abnormal aPTT identified all but two cases by 12 hours; one of these two was identified within 12 hours by leukocytosis. Nine cases of isolated neurotoxicity had a median time of onset after the bite of 4 hours (range, $35 \mathrm{~min}-12 \mathrm{~h}$ ). The combination of serial INR, aPTT and CK tests and repeated neurological examination identified 213 of 222 severe envenoming cases (96\%) by 6 hours and 238 of 240 (99\%) by 12 hours.

Conclusion: Laboratory parameters (INR, aPTT and CK) and neurological reassessments identified nearly all severe envenoming cases within 12 hours of the bite, even in this conservative analysis that assumed normal test results if the test was not done.

MJA 2010; 193: 285-290

Ethics approval has been obtained from 19 human research ethics committees covering all institutions involved in the study.

\section{Study participants}

Patients recruited to ASP from January 2002 to April 2009 were included in this study if at least three sets of laboratory test results were available and the patient was observed in hospital for at least 12 hours after the bite or was admitted to hospital with envenoming. We compared envenomed and nonenvenomed patients, using cases classified as severe envenoming, minor envenoming or non-envenomed.

Severe envenoming was defined as any of the following:

- Venom-induced consumption coagulopathy (VICC): evidence of a complete consumption coagulopathy, indicated by either an undetectable fibrinogen level or a raised D-dimer level (at least 10 times the assay cut-off or $>2.5 \mathrm{mg} / \mathrm{L})$, with an international normalised ratio (INR) $>3.0$.

- Myotoxicity: a creatine kinase (CK) level $>1000 \mathrm{U} / \mathrm{L}$, with myalgia and/or muscle tenderness.

- Neurotoxicity: with either two nerve groups (eg, ocular and bulbar) involved, respiratory muscle paralysis, or requirement for intubation or mechanical ventilation.

- Thrombotic microangiopathy: defined as the presence of intravascular haemolysis on the blood film, thrombocytopenia and an abnormal creatinine level with or without acute renal failure. ${ }^{13}$

Patients with minor envenoming were those with evidence of envenoming but in whom specific treatment or antivenom were rarely required. This included patients with isolated systemic symptoms (at least three of nausea, vomiting, abdominal pain, diarrhoea, diaphoresis and headache), anticoagulant coagulopathy, mild or partial VICC 


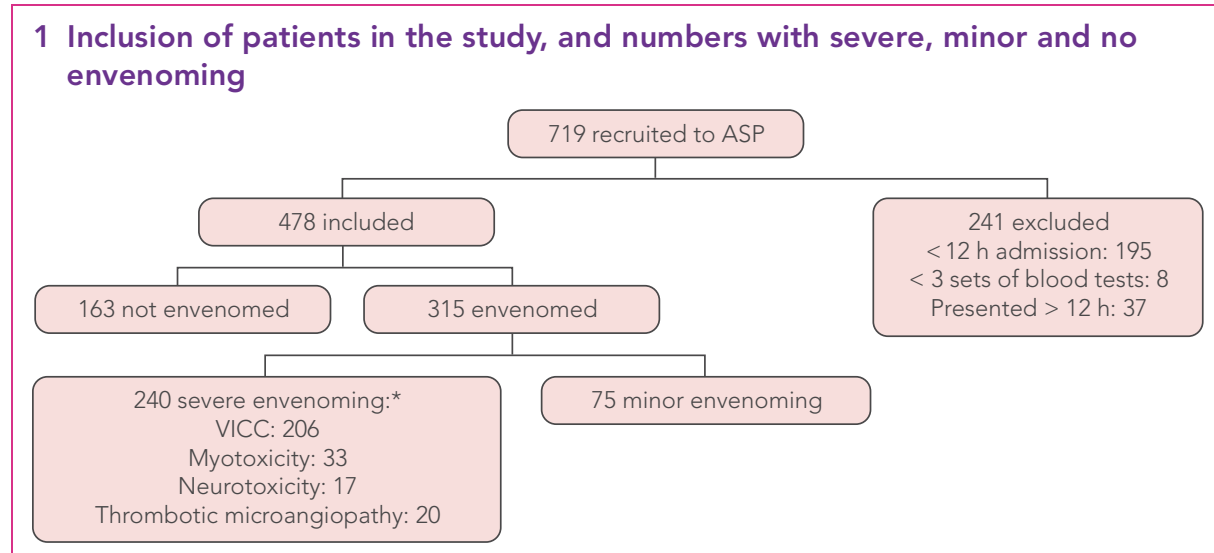

ASP = Australian Snakebite Project. VICC = venom-induced consumption coagulopathy. ${ }^{*}$ Some patients had more than one severe envenoming syndrome, making the sum of the individual syndromes greater than the total number of patients with severe envenoming.

2 Demographic details of snakebite patients*

\begin{tabular}{|c|c|c|c|}
\hline & $\begin{array}{l}\text { Not envenomed } \\
\quad(n=163)\end{array}$ & $\begin{array}{l}\text { Minor envenoming } \\
\qquad(n=75)\end{array}$ & $\begin{array}{l}\text { Severe envenoming } \\
\qquad(n=240)\end{array}$ \\
\hline $\begin{array}{l}\text { Age in years, median } \\
\text { (IQR; range) }\end{array}$ & $36(22-50 ; 1-83)$ & $38(21-56 ; 2-83)$ & $41(25-53 ; 1-87)$ \\
\hline Sex (male) & 107 (66\%) & $53(71 \%)$ & $183(76 \%)$ \\
\hline \multicolumn{4}{|l|}{ State or territory } \\
\hline New South Wales & $38(23 \%)$ & 39 (52\%) & $62(26 \%)$ \\
\hline Queensland & $45(28 \%)$ & $16(21 \%)$ & $68(28 \%)$ \\
\hline Western Australia & $59(36 \%)$ & $9(12 \%)$ & $51(21 \%)$ \\
\hline Victoria & $5(3 \%)$ & $4(5 \%)$ & $25(10 \%)$ \\
\hline South Australia & $8(5 \%)$ & $1(1 \%)$ & $11(5 \%)$ \\
\hline Northern Territory & $7(4 \%)$ & $5(7 \%)$ & $17(7 \%)$ \\
\hline Other & $1(1 \%)$ & $1(1 \%)$ & $6(3 \%)$ \\
\hline \multicolumn{4}{|l|}{ Bite site (if known) } \\
\hline Upper limb & $62(38 \%)$ & $31(41 \%)$ & 107 (45\%) \\
\hline Lower limb & 75 (46\%) & $40(53 \%)$ & $121(50 \%)$ \\
\hline \multicolumn{4}{|l|}{ Snake type ${ }^{\dagger}$} \\
\hline Brown snake & $13(8 \%)$ & $12(16 \%)$ & $116(48 \%)$ \\
\hline Mulga or Collett's snake & $4(2 \%)$ & $9(12 \%)$ & $11(5 \%)$ \\
\hline Death adder & $4(2 \%)$ & $2(3 \%)$ & $10(4 \%)$ \\
\hline Red-bellied black snake & $8(5 \%)$ & $35(47 \%)$ & $4(2 \%)$ \\
\hline Tiger snake group & $3(2 \%)$ & $8(11 \%)$ & 79 (33\%) \\
\hline Taipan & $1(1 \%)$ & $4(5 \%)$ & $11(5 \%)$ \\
\hline Other species & $11(7 \%)$ & 0 & $1(0.4 \%)$ \\
\hline Unknown & $119(73 \%)$ & $5(7 \%)$ & $8(3 \%)$ \\
\hline PBI & $111(68 \%)$ & $55(73 \%)$ & 204 (85\%) \\
\hline Antivenom & $14(9 \%)$ & 44 (59\%) & 238 (99\%) \\
\hline Snake handler & $9(6 \%)$ & $11(15 \%)$ & $36(15 \%)$ \\
\hline
\end{tabular}

$\mathrm{IQR}=$ interquartile range. $\mathrm{PBI}$ = pressure bandages with immobilisation. * Figures are number (\%) unless otherwise indicated. † Determined by a combination of expert snake identification, geographical distribution, clinical spectrum of effects, and venom detection in serum when available. (incomplete consumption coagulopathy characterised by low but detectable fibrinogen level, elevated D-dimer level and a maximum INR <3.0), mild neurotoxicity or myotoxicity not meeting the criteria for severe envenoming.

Non-envenomed patients were those who did not manifest any of these clinical or laboratory features of minor or severe envenoming during their hospital stay of at least 12 hours and did not re-present to the hospital with delayed envenoming.

\section{Data collection}

Information was extracted from the ASP database on: patient demographics; clinical features of envenoming (including time of onset after bite and type of neurological effects); and laboratory test results, including a full blood count, biochemistry, coagulation studies (INR, activated partial thromboplastin time [aPTT], Ddimer and fibrinogen concentrations), and CK level.

\section{Analysis of diagnostic utility of early laboratory test results}

Based on biological plausibility and visual inspection of early serial laboratory test results comparing patients with severe or minor envenoming to non-envenomed patients, only early changes in coagulation test results, CK level, white cell count (WCC) and lymphocyte count were further analysed for diagnostic utility. The cut-off values used to define abnormal results were: INR $>1.2$, aPTT outside the reference range (depending on the individual laboratory), $\mathrm{CK}>250 \mathrm{U} / \mathrm{L}$, and WCC $>11.0 \times 10^{9} / \mathrm{L}$. Based on previous concerns about cases of isolated neurotoxicity occurring in death adder envenoming, ${ }^{9}$ we included a neurological examination in cases of neurotoxicity as part of the analysis.

The primary analysis was of the time from the bite to development of abnormal laboratory test result(s) or neurological examination in patients with severe or minor envenoming, with the conservative assumption that test results were normal if the test was not done (last observation carried forward). For this analysis, we plotted the proportion of envenomed patients who still had normal laboratory test results or neurological examination by time of the test after the snakebite. We then used these plots to estimate the median time until an abnormal laboratory test result or neurological examination occurred. 
In addition, plots were made and the time to development of each abnormal laboratory test result (INR, aPTT, CK and WCC) or neurological examination was estimated for individual envenoming syndromes (VICC, myotoxicity and neurotoxicity). For example, the time to development of an abnormal INR was estimated for patients who developed VICC.

The final analysis was done using three of the above tests - INR, aPTT and CK plus repeated neurological examination within 6 and 12 hours, to determine if severe envenoming could be excluded by normal laboratory test results occurring at these times. A similar analysis was done to determine if minor envenoming could be excluded with the same combination of repeat tests and neurological examination.

For descriptive statistics, median and range, interquartile range (IQR) or 95\% confidence intervals were used for data that were not normally distributed. Statistical and graphical analyses were performed using GraphPad Prism version 5.03 for Windows (GraphPad Software, San Diego, Calif, USA).

\section{RESULTS}

There were 478 patients in three groups: 240 had severe envenoming, 75 had minor envenoming and 163 were not envenomed (Box 1, Box 2). PBI were used on 370 patients $(77 \%)$ at some time.

\section{Venom-induced consumption coagulopathy}

VICC occurred in 206 patients, including 174 who had VICC alone and 16 who had VICC with thrombotic microangiopathy. The remainder had VICC with either neurotoxicity (6) or myotoxicity (8), or both (2).

In 178 of the 206 patients (86\%), the INR was abnormal (>1.2) on the first set of tests using blood samples taken a median of 1 hour and 36 minutes after the bite (range, $20 \mathrm{~min}-11$ h $30 \mathrm{~min}$ ) (Box 3). The remaining 28 patients had a normal INR recorded before VICC developed (including one patient who had a second normal INR at 3 hours and 45 minutes after the bite). For these 28 patients, the median time after the bite to the normal INR was 1 hour (range, $7 \mathrm{~min}-3 \mathrm{~h} 45 \mathrm{~min}$ ) and the median time after the bite to the first abnormal INR was 4 hours and 17 minutes (range, 1 h 55 min $10 \mathrm{~h} 30 \mathrm{~min})$. In seven patients where the time from the bite to the first abnormal INR

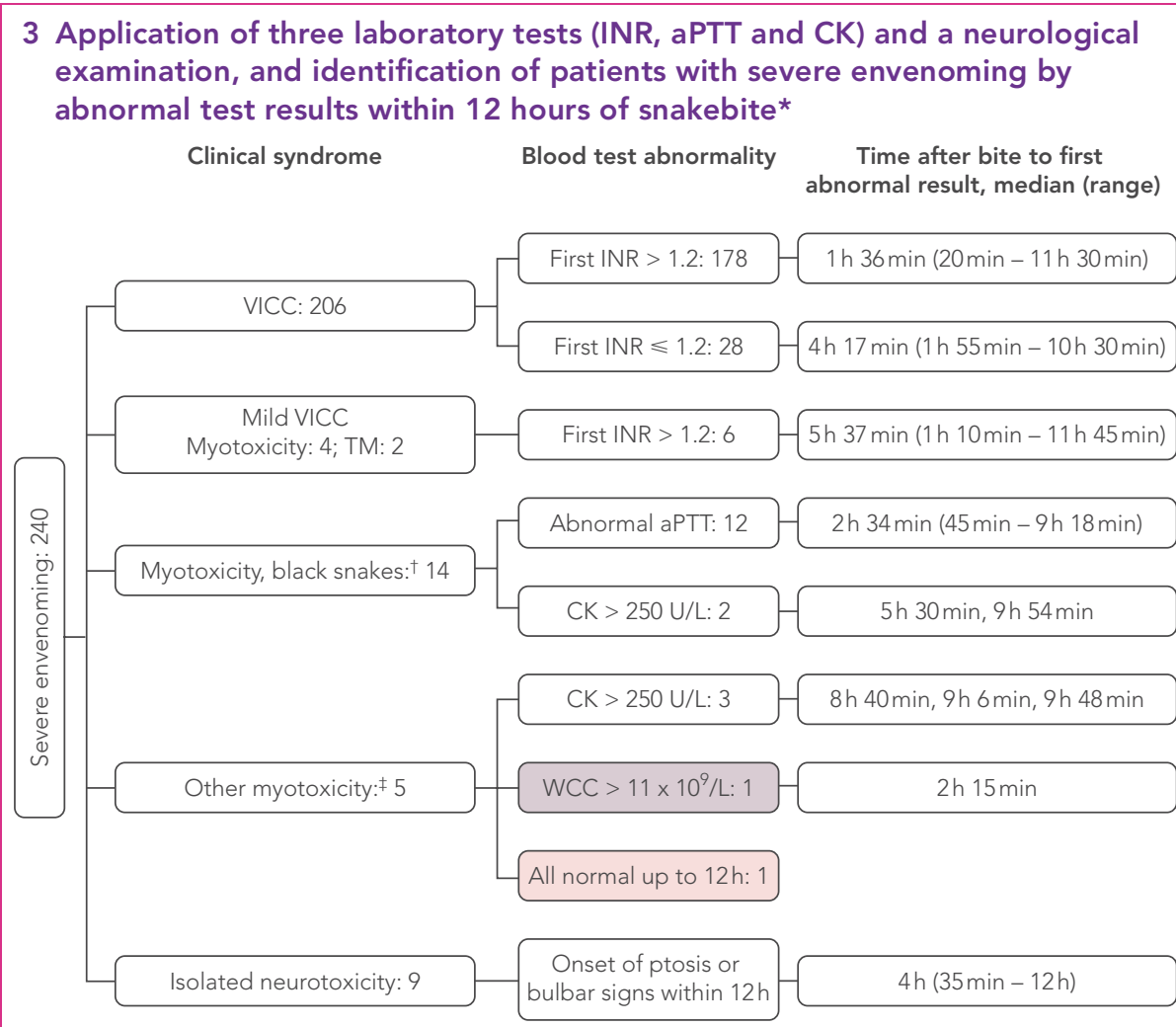

$\mathrm{VICC}=$ venom-induced consumption coagulopathy. $\mathrm{TM}=$ thrombotic microangiopathy. INR = international normalised ratio. aPTT = activated partial thromboplastin time. $\mathrm{CK}=$ creatine kinase. $\mathrm{WCC}=$ white cell count. * Shaded boxes represent the two cases not identified by this combination of tests within 12 hours (see text). $\dagger$ Pseudechis spp. ¥ Myotoxicity in the absence of coagulopathy.

was greater than 6 hours, this reflected a delay of 4 or more hours between the first (normal) INR and the second (abnormal) INR. For all patients with VICC, the median time to an abnormal INR was 1.8 hours (range, $20 \mathrm{~min}-11 \mathrm{~h} 30 \mathrm{~min}$ ) (Box 4, A).

Of the 28 patients whose first INR was normal, 22 had other parameters measured at the same time: 12 of these had an abnormal D-dimer (> assay cut-off) or low fibrinogen $(<1.5 \mathrm{~g} / \mathrm{L})$ level. Twenty-five of the 28 patients (89\%) with an initial normal INR had PBI at the time, compared with 149 of the 178 patients (84\%) whose first INR was abnormal despite PBI. The relative risk for an initial normal INR with PBI was 0.65 (95\% CI, 0.21-2.00; $P=0.58$ ).

Of 31 patients with partial VICC, 26 had an abnormal INR within 12 hours. Of the remaining five, two had an abnormal aPTT within 12 hours; one had myotoxicity with CK > 250 U/L within 12 hours; and two never had an abnormal INR, and identification of their partial VICC was based on a low fibrinogen or high D-dimer level.

There were 20 patients with thrombotic microangiopathy, and all had preceding evi- dence of VICC, with an abnormal INR within 12 hours. Seventeen of these patients had VICC and three had partial VICC.

\section{Myotoxicity}

Myotoxicity occurred in 33 patients with severe envenoming who were bitten by mulga snakes (Pseudechis australis) (8), redbellied black snakes (Pseudechis porphyriacus) (4), Collett's snake (Pseudechis colletti) (3), snakes of the tiger snake group (Notechis spp, Tropidechis carinatus) (14), taipans (Oxyuranus scutellatus) (2), a death adder (Acanthophis praelongus) (1) and a sea snake (1).

The median maximum $\mathrm{CK}$ level was 5390 U/L (range, 1054-203 $110 \mathrm{U} / \mathrm{L}$ ) in patients with myotoxicity. An abnormal CK level had occurred in 10 of the 33 patients (30\%) at 6 hours and in 24 patients (73\%) at 12 hours. An abnormal aPTT had occurred in 20 patients $(61 \%)$ at 6 hours and in 25 patients (76\%) at 12 hours.

Two patients with myotoxicity did not have an abnormal CK level or aPTT within 12 hours (Box 3). One of these patients had a normal CK level but an elevated WCC at 2 hours and 15 minutes, and the next sam- 
ple was not taken until 33 hours and 30 minutes after the bite, which then had a CK level of $4770 \mathrm{U} / \mathrm{L}$. The second patient had a CK level of $220 \mathrm{U} / \mathrm{L}$ at 4 hours and no further testing until 20 hours after the bite, with a CK level in that sample of $691 \mathrm{U} / \mathrm{L}$. This patient developed myotoxicity with localised myalgia and a CK level peaking at $12028 \mathrm{U} / \mathrm{L}$ at 46 hours after the bite, with ptosis and diplopia but no VICC.

For patients with myotoxicity, the median time to an abnormal CK level was 9.1 hours (range, $45 \mathrm{~min}$ - $33 \mathrm{~h} 30 \mathrm{~min}$ ), and median time to an abnormal aPTT was 4.2 hours (range, $45 \mathrm{~min}$ - 9 h $30 \mathrm{~min}$ ) (Box 4, A).

Of 120 non-envenomed patients who had their CK level measured in the first 6 hours, 18 (15\%) had an abnormal CK level. Of these 18, the CK level decreased on the next blood test for 13, and on the second subsequent blood test for three. It was not retested for two patients. This compared with an increase in CK level on the next blood test in all cases of myotoxicity.

\section{Isolated neurotoxicity}

There were nine cases of isolated severe neurotoxicity, and all nine were from death adder bites. The median time of onset of neurotoxicity after the bite was 4 hours (range, $35 \mathrm{~min}$ - $12 \mathrm{~h}$ ) (Box 4, A). Eight patients had ptosis, and the ninth had bulbar weakness. Three patients who developed respiratory muscle paralysis and required mechanical ventilation had evidence of neurotoxicity after 35 minutes, 3 hours, and 4 hours, respectively.

\section{Leukocytosis}

An abnormal WCC developed in 192 of the 240 patients with severe envenoming (80\%), and the median time to an abnormal WCC in these patients was 5.7 hours (range, 30 min - 26 h 45 min) (Box 4, A). Leukocytosis occurred in similar numbers of mildly and severely envenomed patients, and only identified one case of severe envenoming not identified by the other three tests within 12 hours in this study (Box 3). Patients with brown snake (Pseudonaja spp) bites were less likely to have leukocytosis occur at any time, compared with patients with bites from the tiger snake group (Notechis spp and T. carinatus) or red-bellied black snakes ( $P$. porphyriacus).

\section{Proposed algorithm}

Using a combination of three laboratory tests (INR, aPTT and CK) and repeated

\section{Plots of the proportion of envenomed patients with normal laboratory test results versus time after bite}

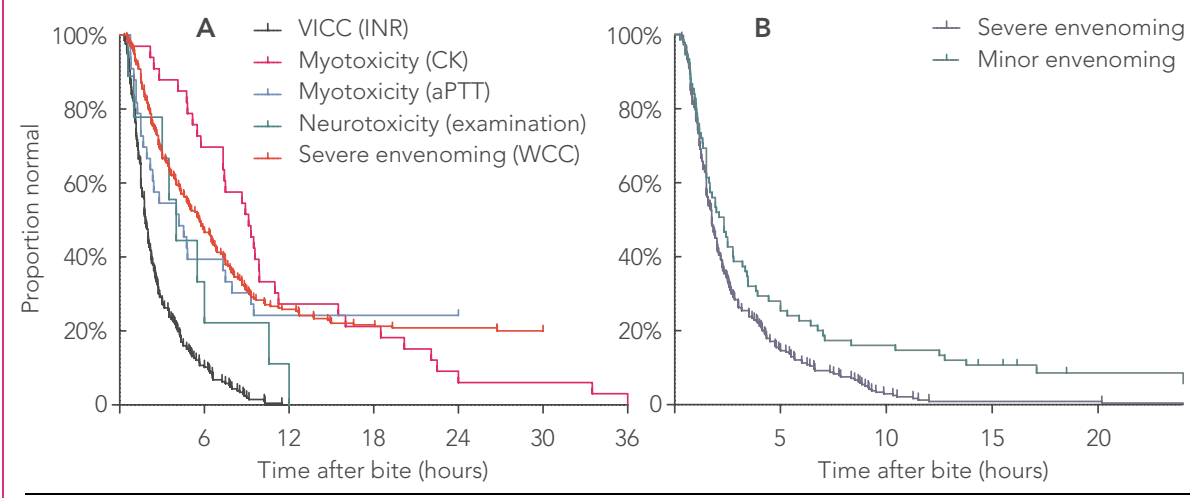

A: Proportions of patients with: a normal international normalised ratio (INR) in venom-induced consumption coagulopathy (VICC); a normal creatine kinase (CK) level in myotoxicity; a normal activated partial thromboplastin time (aPTT) in myotoxicity; a normal neurological examination in isolated neurotoxicity; and a normal white cell count (WCC) in severe envenoming.

B: Proportions of patients with normal results of INR, aPTT and CK tests or a normal neurological examination, in severe envenoming and minor envenoming.

neurological examination, 238 of the 240 patients with severe envenoming (99\%) had an abnormal laboratory test result or evidence of neurotoxicity by 12 hours (Box 3 and Box 4, B). Both patients who did not have abnormal test results within 12 hours had myotoxicity and did not have laboratory tests performed at 6 or 12 hours, when it was likely their CK levels would have been elevated. There were 222 patients with severe envenoming who presented within 6 hours of the bite, and 213 of these (96\%) had documented abnormal laboratory test results at 6 hours. Applying the same laboratory criteria to the minor envenoming cases, 64 of the 75 patients ( $85 \%$ ) had abnormal results within 12 hours (Box 4, B). The median time to an abnormality (laboratory test result or neurological examination) was 1.75 hours (range, $20 \mathrm{~min}$ - 33 h $30 \mathrm{~min}$ ) for severe envenoming and 2.1 hours (15 min - 30 h $45 \mathrm{~min}$ ) for minor envenoming.

\section{DISCUSSION}

This study suggests that the combination of tests for INR, aPTT and CK level and serial neurological examinations is able to reliably detect envenomed patients within 12 hours. No single test was sufficiently useful to exclude severe envenoming within 12 hours, and a combination of the three tests, which are relevant to different envenoming syndromes, was most appropriate. The study supports an observation period of 12 hours for suspected snakebite, with repeat laboratory testing and neurological assess- ments performed on admission and at 6 hours and 12 hours after the bite (Box 5).

Although PBI were used for most patients and may have delayed the onset of envenoming in some, the majority had evidence of envenoming on admission despite the use of PBI. Current recommendations are that repeat blood tests should be performed 1 hour after PBI removal because envenoming can develop rapidly after removal of effective PBI. ${ }^{14}$

Most literature suggests that only 5\% to $10 \%$ of snakebite patients develop severe envenoming, ${ }^{1,3,8,15-19}$ so much of the emergency department or hospital workload is the assessment of suspected snakebite to exclude severe envenoming. This study provides an approach to assessing these patients based on a large number of severely envenomed patients. Coagulation studies were the most useful early laboratory parameters (Box 4, A) because the major clinical syndrome was VICC. The aPTT was an early detector of myotoxicity because of the presence of an anticoagulant coagulopathy in black snake envenoming and of VICC in tiger snake and taipan envenoming - the snake types that most often cause myotoxicity. CK level was not a good early detector of myotoxicity and in some cases took over 12 hours to become abnormal in affected patients, although this was often due to delayed repeat CK testing (Box 4, A).

The WCC and lymphocyte count were less useful as early detectors of envenoming because, unlike VICC and myotoxicity, where the INR and CK level, respectively, must become abnormal by definition, this is not the case for WCC, which remained 


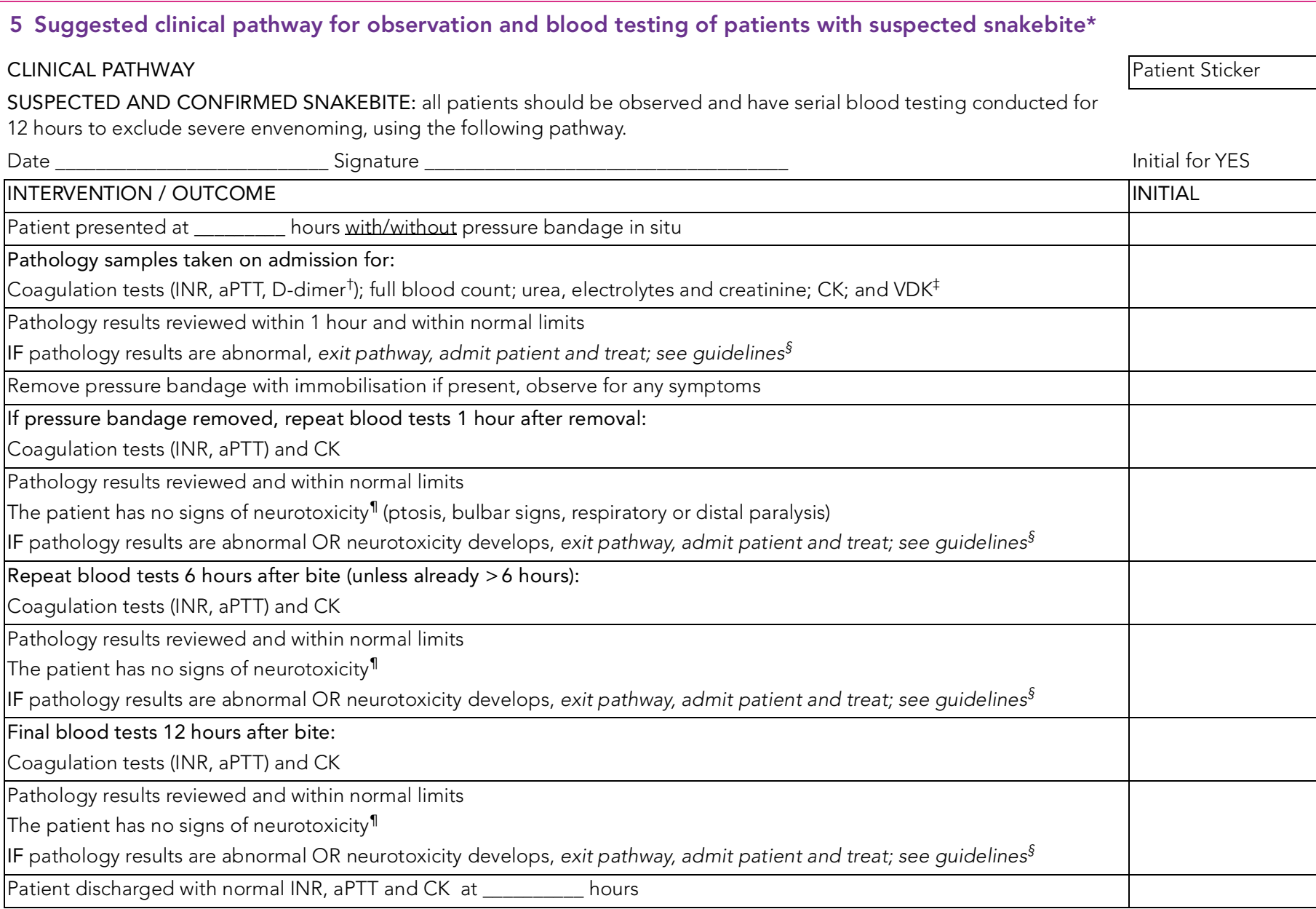

INR = international normalised ratio. aPTT = activated partial thromboplastin time. CK = creatine kinase. VDK= Venom Detection Kit (CSL Ltd, Melbourne, Vic). *Some geographical regions with different venomous snake fauna are not well represented in our dataset, and local modification of this pathway may be needed to account for differences in snake fauna. †D-dimer test may need repeating if it is moderately elevated (ie, greater than assay cut-off but less than 10 times the cut-off) because this is more likely to be a false positive. ¥A bite-site swab should be collected and stored, but only tested if there is envenoming. § Applicable guidelines at treatment site. I Signs of neurotoxicity can be subtle and it is important to include both looking for ptosis and assessing for fatigue (eg, eyelid droop from failure to maintain an upward gaze).

normal for the duration of observation in some patients with severe envenoming. In this study, an abnormal WCC only identified one further case of severe envenoming that was not detected within 12 hours by the other tests (Box 3), and it is likely that if a CK level had been tested at 12 hours in this patient, it would have been abnormal. The WCC did not reflect severity of envenoming and was more often raised in cases of redbellied black snake envenoming, which did not commonly cause severe envenoming, compared with brown snake envenoming, which did commonly cause severe envenoming with VICC. The WCC was therefore not included with the three laboratory tests we selected to identify whether envenoming had occurred.

There are a number of limitations of this study, including a greater proportion of envenomed patients than would be repre- sentative of normal practice. However, our focus was on identifying early development of an abnormal blood test result to indicate envenoming ("sensitivity"), which is unlikely to be affected by this. However, using our suggested approach means that some patients who do not have envenoming will be kept in hospital if they have an abnormal laboratory test result. This most commonly occurs for CK; $15 \%$ of nonenvenomed patients in this study who had their CK level measured within 6 hours had an abnormal result. Using our approach, these patients would be observed in hospital until it is clear they do not have venominduced myotoxicity and their CK level is seen to be decreasing rather than increasing over time.

The approach to specific treatment once an abnormal laboratory test result occurs is complex and beyond the scope of this study, which simply answers the initial assessment question of whether the patient is envenomed and, importantly, when clinicians can be confident that envenoming will not occur.

Another problem is that there were few cases of myotoxicity (33) and isolated neurotoxicity (9) in our study. These syndromes are a major source of delayed or missed envenoming, so larger studies of these groups are needed to confirm our approach in such cases. In addition, modification to the assessment pathway proposed in Box 5 may be needed for geographical regions with venomous snake fauna not well represented in our dataset.

Additionally, although a standardised series of blood tests was suggested for all patients recruited to ASP (at admission, 1 hour after release of first aid, and 6 hours and 12 hours after the bite), this did not 
occur in many cases. This meant that we undertook a conservative analysis because we assumed that a test result was normal until the first abnormal test result occurred. In the case of VICC, the latest normal INR was at 3 hours and 45 minutes, compared with the latest abnormal INR at 10 hours and 30 minutes. This apparent delay of over 10 hours until an abnormal INR is more likely due to a delay before the next INR test was done, rather than the INR actually remaining normal for an additional 6 hours.

Another limitation was the definition of non-envenoming, which was based on patients having no clinical effects and normal blood test results on discharge, which may have been soon after 12 hours in some cases. There is a possibility that some of these patients developed envenoming after discharge. However, Box 4, B suggests that the proportion of patients developing abnormal test results after 12 hours would be negligible. In addition, if patients represented to hospital with delayed onset of envenoming, this would have been identified in the ASP database by additional laboratory tests being ordered.

Further, we used a definition of "clinical" envenoming, based on whether treatment was required, and venom concentrations were not measured to confirm envenoming. Although the presence of circulating venom may be the literal interpretation of envenoming, ultimately a clinical definition is more useful, and this is why we focused on envenoming defined by clinical severity.

In conclusion, in this series of snakebite patients, the combination of INR, aPTT and CK level, together with repeated neurological assessments, detected all but two cases of severe envenoming within 12 hours. Most cases of severe envenoming (96\%) were identified at 6 hours using this combination of tests. The results of this study can be used to develop potential clinical pathways, such as that shown in Box 5. However, such protocols need to be tested in a prospective study of patients with suspected snakebite.

\section{ACKNOWLEDGEMENTS}

This article was written on behalf of the ASP clinical investigators who recruited patients to the study: Michael Taylor (Albury Base Hospital), Yusuf Nagree (Armadale Hospital), Fergus Kerr (Austin Hospital), Conrad Macrokanis (Broome Hospital), Garry Wilkes (Bunbury Hospital), Robert Bonnin, Richard Whitaker and Lambros Halkidis (Cairns Base Hospital), Geoff Isbister (Calvary Mater Newcastle Hospital), Nicholas Buckley (Canberra Hospital), Alan Tankel (Coffs Harbour Base Hospital), Randall Greenberg (Dubbo Base Hospital), Mark
Webb (Flinders Medical Centre), Rod Ellis (Fremantle Hospital and Rockingham Hospital), David Spain and Graham Ireland (Gold Coast Hospital), Mark Miller (John Hunter Hospital), Chris Gavaghan (Lismore Base Hospital), Anna Holdgate (Liverpool Hospital), Todd Fraser (Mackay Hospital), Mark Coghlan (Nambour Hospital), Colin Page (Princess Alexandra Hospital), Peter Thompson (Rockhampton Hospital), Julian White (Royal Adelaide Hospital and Women's and Children's Hospital, Adelaide), Tanya Gray (Royal Children's Hospital, Brisbane), Bart Currie (Royal Darwin Hospital), Justin Yeung, Simon Brown and David McCoubrie (Royal Perth Hospital), Mark Little and Ovidiu Pascu (Sir Charles Gairdner Hospital), Nick Ryan (Tamworth Hospital), Ben Close (Townsville Hospital), Shane Curran (Wagga Wagga Base Hospital), Naren Gunja (Westmead Hospital); and the ASP laboratory investigators including Margaret O'Leary, Sarah Just and Vaughan Williams. We also acknowledge the many referrals from poison information centres and clinical toxicologists, and help of the many other nurses, doctors and laboratory staff in recruiting patients and collecting samples. This study was supported in part by National Health and Medical Research Council (NHMRC) Project Grant 490305, and Simon Brown and Geoff Isbister are both funded by NHMRC Clinical Career Development Awards.

\section{COMPETING INTERESTS}

Julian White has received travel assistance from CSL Ltd (producer of antivenom) to attend international meetings as an invited speaker.

\section{AUTHOR DETAILS}

Graham Ireland, MB BS, Senior Registrar ${ }^{1}$ Simon GA Brown, FACEM, PhD, Professor ${ }^{2}$ Nicholas A Buckley, BMed, FRACP, MD, Professor $^{3}$

Jeff Stormer, RN, Research Nurse ${ }^{4}$

Bart J Currie, MB BS, FRACP, Director ${ }^{5}$

Julian White, MB BS, MD, Director ${ }^{6}$

David Spain, MB BS, FACEM, Senior Staff

Specialist ${ }^{1}$

Geoffrey K Isbister, BSc, FACEM, MD, Senior Research Academic ${ }^{4,5,7}$

1 Emergency Department, Gold Coast Hospital, Gold Coast, QLD.

2 Centre for Clinical Research in Emergency Medicine, Western Australian Institute for Medical Research and Emergency Medicine, Royal Perth Hospital, University of Western Australia, Perth, WA.

3 Prince of Wales Hospital Medical School, University of New South Wales, Sydney, NSW.

4 Department of Clinical Toxicology and Pharmacology, Calvary Mater Newcastle Hospital, Newcastle, NSW.

5 Tropical and Emerging Infectious Diseases, Menzies School of Health Research, Charles Darwin University, Darwin, NT.

6 Department of Toxinology, Women's and Children's Hospital, Adelaide, SA.

7 Discipline of Clinical Pharmacology, University of Newcastle, Newcastle, NSW.

Correspondence: geoff.isbister@gmail.com

\section{REFERENCES}

1 Isbister GK, Currie BJ. Suspected snakebite: one year prospective study of emergency department presentations. Emerg Med (Fremantle) 2003; 15: 160-169.

2 Jelinek GA, Breheny FX. Ten years of snake bites at Fremantle Hospital. Med J Aust 1990; 153: 658-661.

3 Hughes A. Observation of snakebite victims: is twelve hours still necessary? Emerg Med (Fremantle) 2003; 15: 511-517

4 Gavaghan CF, Sparkes G. Delayed myotoxicity in snake envenoming by the tiger snake group. Emerg Med (Fremantle) 2003; 15: 497-499.

5 Hood VL, Johnson JR. Acute renal failure with myoglobinuria after tiger snake bite. Med J Aust 1975; 2: 638-641.

6 Furtado MA, Lester IA. Myoglobinuria following snakebite. Med J Aust 1968; 1: 674-676.

7 White J. Elapid envenomation. In: Covacevich J, Davie P, Pearn J, editors. Toxic plants and animals: a guide for Australia. 1st ed. Brisbane: Queensland Museum, 1987.

8 Mead HJ, Jelinek GA. Suspected snakebite in children: a study of 156 patients over 10 years. Med J Aust 1996; 164: 467-470.

9 Currie BJ. Snakebite in Australia: moving from anecdotes to prospective studies. Emerg Med (Fremantle) 2003; 15: 406-408.

10 Isbister GK, Brown SG, MacDonald E, et al. Current use of Australian snake antivenoms and frequency of immediate-type hypersensitivity reactions and anaphylaxis. Med J Aust 2008; 188: 473-476.

11 Isbister GK, Duffull SB, Brown SG; ASP Investigators. Failure of antivenom to improve recovery in Australian snakebite coagulopathy. QJM 2009; 102: 563-568.

12 Isbister GK, Williams V, Brown SG, et al. Clinically applicable laboratory end-points for treating snakebite coagulopathy. Pathology 2006; 38: 568-572.

13 Isbister GK, Little M, Cull G, et al. Thrombotic microangiopathy from Australian brown snake (Pseudonaja) envenoming. Intern Med J 2007; 37: 523-528.

14 Pearn J, Morrison J, Charles N, Muir V. First-aid for snake-bite: efficacy of a constrictive bandage with limb immobilization in the management of human envenomation. Med J Aust 1981; 2: 293-295.

15 Barrett R, Little M. Five years of snake envenoming in far north Queensland. Emerg Med (Fremantle) 2003; 15: 500-510.

16 Munro JG, Pearn JH. Snake bite in children: a five year population study from South-East Queensland. Aust Paediatr J 1978; 14: 248-253.

17 Jamieson R, Pearn J. An epidemiological and clinical study of snake-bites in childhood. Med J Aust 1989; 150: 698-702.

18 White J. Patterns of elapid envenomation and treatment in South Australia. Toxicon 1983; 21 Suppl 3: 489-491.

19 Jelinek GA, Hamilton T, Hirsch RL. Admissions for suspected snake bite to the Perth adult teaching hospitals, 1979 to 1988. Med J Aust 1991; 155: 761-764.

(Received 29 Jul 2009, accepted 2 Jul 2010) 